\title{
Re-acquired Walking is Possible by the Rehabilitation Treatment with Using Device
}

\author{
Shigeo Takizawa \\ The 21st Century Rehabilitation Research Society, director
}

Orthopedic doctors and an author published an article of the specific and kinetic rehabilitation method in the journal of the Japanese Clinical Orthopaedic Association meeting for the future ${ }^{1)}$. We clarified that $30 \%$ of the elderly who were bedridden by sequela of fracture and/or cerebrovascular disorder the became a walk possible in the paper. It is a fact whose 59 persons' walk was attained from the 193 bedridden elderly. It means that $30 \%$ of them became a walk possible, as a result of performing Takizawa Method rehabilitation. The subjects were unable to walk at the time of hospitalized; the oldest was 99 years old, youngest was 47 years old, and the average age was 81 years old, and 137 women and 56 men.

The 126 patients could train at the rehabilitation training room, and 67 did bedside training. 59 subjects re-acquired walking and itemized; 9 patients could walk independently, 7 could do crutch walking, 3 could do with a four wheels' walker, 11 could do with a four wheels' walker with sleds, and 29 could do in parallel bars. If this is applied to the 2.3 million bedridden elderly currently assumed in 2025 , the total of the re-acquired walking will amount to 690,000 .

We organized the 21st Century Rehabilitation Research Society and enforced research in order to continue an independent life even if there was a sequela of fracture or cerebrovascular disorder, to get for people an old age in comfort, and to enable them to live richly based on this fact in the aged society.

The total population of Japan will start to decrease in 2007 , and the 65 or older elderly will be one of four people in 2020. The 4 million children aged 14 and lower will decrease from the present and will be the half of the number of elderly. The study group of the Economic Planning Agency (that time) predicted that national burden rate went up from $35.8 \%$ of the present to $51.1 \%$ after 30 years in 2025 . There is much other pessimistic prediction of the national income $3 / 4$ disappearing, and they advocated that the existing systems, such as finances and a pension collapse if it is neglected without changing the present structure.

We tried to develop devices which can enforce the test for scientific and medical assessment and proposed a field test to clarify the outline of the evaluation for every case in order to prevent the Aging Crisis.

Keywords: Aging, decrease in the birthrate, motivative exercise, cerebrovascular disorder sequela, Takizawa Method, reacquired walking

Reference

1) Hideo Kijima, Kenji lizuka, Shigenobu Imai, Toshiaki Kato, Hitomi Watanabe, Shiro Kanai, Kyoko Takizawa, and Shigeo Takizawa, The rehabilitation and the related training devices which we have recommended, Clinical Orthopaedic Association Journal, 23 (58) (1998), pp186-191. (JP)

Notice and Reference;

This is an abstract of Japanese article of the republished. Full Text (JP) on BRJ (2017-1) p. 5-8, https://doi.org/10.3774/brj.2017.5

It is a re-publication of the Jiji Press Welfare 4651, pp2-4, 6 June 1998. (JP), which was permitted to publish by the editor of the Journal at the time of publication. 